\title{
LRRK2 Modulates Vulnerability to Mitochondrial Dysfunction in Caenorhabditis elegans
}

\author{
Shamol Saha, ${ }^{1}$ Maria D. Guillily, ${ }^{1 \star}$ Andrew Ferree, ${ }^{1 \star}$ Joel Lanceta, ${ }^{1}$ Diane Chan, ${ }^{1}$ Joy Ghosh,${ }^{2}$ Cindy H. Hsu, ${ }^{1}$ \\ Lilach Segal, ${ }^{1}$ Kesav Raghavan, ${ }^{1}$ Kunihiro Matsumoto, ${ }^{5}$ Naoki Hisamoto, ${ }^{5}$ Tomoki Kuwahara, ${ }^{6}$ Takeshi Iwatsubo, ${ }^{6}$ \\ Landon Moore, ${ }^{3}$ Lee Goldstein, ${ }^{2}$ Mark Cookson, ${ }^{7}$ and Benjamin Wolozin ${ }^{1,4}$ \\ Departments of ${ }^{1}$ Pharmacology, ${ }^{2}$ Psychiatry, ${ }^{3}$ Medicine, and ${ }^{4}$ Neurology, Boston University School of Medicine, Boston, Massachusetts $02118,{ }^{5}$ Department \\ of Molecular Biology, Institute for Advanced Research, Nagoya University, Nagoya 464-8602, Japan, ${ }^{6}$ Department of Neuropathology and Neuroscience, \\ Graduate School of Pharmaceutical Science, University of Tokyo, Tokyo 113-0033, Japan, and ${ }^{7}$ Cell Biology and Gene Expression Unit, Laboratory of \\ Neurogenetics, National Institute of Aging, Bethesda, Maryland 20892
}

Mutations in leucine-rich repeat kinase 2 (LRRK2) cause autosomal-dominant familial Parkinson's disease. We generated lines of Caenorhabditis elegans expressing neuronally directed human LRRK2. Expressing human LRRK2 increased nematode survival in response to rotenone or paraquat, which are agents that cause mitochondrial dysfunction. Protection by G2019S, R1441C, or kinase-dead LRRK2 was less than protection by wild-type LRRK2. Knockdown of lrk-1, the endogenous ortholog of LRRK2 in C. elegans, reduced survival associated with mitochondrial dysfunction. C. elegans expressing LRRK2 showed rapid loss of dopaminergic markers (DAT::GFP fluorescence and dopamine levels) beginning in early adulthood. Loss of dopaminergic markers was greater for the G2019S LRRK2 line than for the wild-type line. Rotenone treatment induced a larger loss of dopamine markers in C. elegans expressing G2019S LRRK2 than in C. elegans expressing wild-type LRRK2; however, loss of dopaminergic markers in the G2019S LRRK2 nematode lines was not statistically different from that in the control line. These data suggest that LRRK2 plays an important role in modulating the response to mitochondrial inhibition and raises the possibility that mutations in LRRK2 selectively enhance the vulnerability of dopaminergic neurons to a stressor associated with Parkinson's disease.

\section{Introduction}

Parkinson's disease (PD) is the most common neurodegenerative movement disorder. At least nine different genes are known to cause familial PD. Functional studies of PD-related genes implicate dysfunction of mitochondria, protein aggregation, and the stress response in the pathophysiology of PD. Autosomaldominant mutations in leucine-rich repeat kinase 2 (LRRK2) are associated with both familial and late-onset PD (Paisán-Ruíz et al., 2004; Zimprich et al., 2004). The G2019S mutation is the most common mutation and is present in $\sim 40 \%$ of familial and sporadic PD in Arab samples from North Africa, 30\% of familial

\footnotetext{
Received May 14, 2009; revised June 11, 2009; accepted June 17, 2009.

This work was supported by National Institutes of Health (NIH)-National Institute of Environmental Health Sciences Grant ES015567, NIH-National Institute of Neurological Disorders and Stroke Grant NS060872, the Alzheimer Association, and the Michael J. Fox Foundation (all to B.W.). This research was also supported in part by the Intramural Research Program of the NIH, National Institute on Aging, Project 1201 AG000953 (awarded to M.C.) Some nematode strains used in this work were provided by the Caenorhabditis Genetics (enter, which is funded by the NIH National Center for Research Resources. We thank the following individuals for their invaluable help: Shohe Mitani (Tokyo Women's Medical College, Tokyo, Japan) for assisting with acquisition of the DAT::GFP nematodes; Scott Clark (Skirball Institute, New York University, New York, NY) for provision of the strains SK4005, n765ts, and the pSK1 plasmid; Anne Hart (Center for Cancer Research, Massachusetts General Hospital, Charlestown, MA) for her valuable advice; and Bryan Yamamoto (Department of Pharmacology, Boston University, Boston, MA) for advice on dopamine measurements.

*M.G. and A.F. contributed equally to this work.

Correspondence should be addressed to Dr. Benjamin Wolozin, Department of Pharmacology, Boston University

School of Medicine, 72 E. Concord Street, R614, Boston, MA 02118-2526. E-mail: bwolozin@bu.edu.

L. Moore's present address: Department of Biology, University of Southern Indiana, Evansville, IN 47712.

DOI:10.1523/JNEUROSCI.2281-09.2009

Copyright $\odot 2009$ Society for Neuroscience $\quad$ 0270-6474/09/299210-09\$15.00/0
}

PD in Ashkenazi Jewish populations, and up to 3\% of sporadic PD in Europe and North America (Lesage et al., 2006; Ozelius et al., 2006).

LRRK2 is a member of the ROCO protein family and contains multiple domains, including a leucine-rich repeat domain, Roc GTPase, COR (C-terminal of Ras) of unknown function, kinase domain, and WD40-repeat domain at the $\mathrm{C}$ terminal (Zimprich et al., 2004). LRRK2 is present in the cytoplasm and is also associated with membranes, including those of mitochondria, endoplasmic reticulum, and synaptic vesicles (Biskup et al., 2006). Recent studies demonstrate that mutations, such as G2019S, lead to increased LRRK2 autophosphorylation and kinase activity (West et al., 2005; Greggio et al., 2006). Transient expression of mutant forms of LRRK2 in neurons causes cell death, and the death appears to require LRRK2 kinase activity. Expressing mutant LRRK2 in neurons also decreases neurite length in rodents (MacLeod et al., 2006). Mammals have a close homolog of LRRK2, termed LRRK1. This protein (LRRK1) also has kinase and GTPase activity but is less toxic than LRRK2 after transient transfection (Greggio et al., 2007). The ortholog of LRRK1 and 2 in Caenorhabditis elegans is lrk-1. In lrk-1 deletion mutants, synaptic vesicle proteins are mislocalized in a small subset of neurons (Sakaguchi-Nakashima et al., 2007). The mechanism by which these changes occur is not known. Dysfunction of mitochondria is implicated in the pathophysiology of PD (Ved et al., 2005; Schapira, 2008). For instance, $\alpha$-synuclein is associated with dysfunction of mitochondria and the endoplasmic reticulum (Chan- 
dra et al., 2004; Cooper et al., 2006; Schapira, 2008). Parkin, PINK-1, and DJ-1, which are genes associated with familial PD, are also associated with mitochondrial function (Greene et al., 2003; Yang et al., 2006; Exner et al., 2007; Plun-Favreau et al., 2007). These observations raise the possibility that LRRK2 also modulates mitochondrial function.

We generated lines of $C$. elegans expressing human wild-type (WT) LRRK2 and the mutants G2019S, R1441C, and two kinasedead (KD) variants of LRRK2 (KD and R1441C/KD), to investigate how LRRK2 affects biological functions linked to PD. We report that WT LRRK2 protects against mitochondrial dysfunction, but disease-related mutants of LRRK2 appear to produce responses that range from less protection to overt enhancement of toxicity.

\section{Materials and Methods}

Chemicals. All chemicals were purchased from Sigma unless otherwise indicated. Bacterial agar, peptone, and Luria broth (LB) media/agar were purchased from American Bioanalytical.

Plasmids. V5-tagged WT and mutant LRRK2 cDNAs were obtained from mammalian Gateway entry vectors (Invitrogen), generated and described by Greggio et al. (2006). LRRK2 constructs were transferred from these Gateway entry vectors to a Gateway-compatible C. elegans expression vector, pSybDEST, that was derived from pPD95.76 (Fire vector) by adding a ccdB-containing fragment with integration acceptor sites into XhoI site of pPD95.76. The directional gene transfers were performed via entry vectors as described in the Gateway cloning protocol. All interference RNA (RNAi) vectors were purchased from Open Biosystems.

C. elegans culture. Standard methods were used for culturing C. elegans on nematode growth medium (NGM) agar (Brenner, 1974; Wood, 1988). The lrk-1 [km17] line has a deletion that removes the kinase and WD40 domains; generation and characterization of 1rk-1 [km17] was described previously (Sakaguchi-Nakashima et al., 2007). The SK4005 [zDIs5] (Mec-4::GFP) I; lin-15 (+) line expresses green fluorescent protein (GFP) driven by the C. elegans mechanosensory Mec4 promoter (Suzuki et al., 2003) and was generously provided by Scott Clark (Skirball Institute, New York, NY). The lin-15 (+) line was generated by complementation of the temperature-sensitive lin-15 (n765ts) mutation, as described for the LRRK2 lines below (Clark et al., 1994).

Generating transgenic C. elegans lines. LRRK2 transgenic nematodes were created by coinjecting a mixture of DNAs containing $50 \mathrm{ng} / \mu \mathrm{l}$ plasmid pSyb-LRRK2 or pSyb-LRRK2 mutants along with WT lin-15 plasmid (pSK1; $20 \mathrm{ng} / \mu \mathrm{l})$ and sonicated $C$. elegans genomic DNA (20 $\mathrm{ng} / \mu \mathrm{l})$. This mixture of DNA was injected into young adults of lin-15 strain (n765ts), grown at $15^{\circ} \mathrm{C}$ (Clark et al., 1994). Injected nematodes were grown at $20^{\circ} \mathrm{C}$ and MUVless progenies, complemented with WT lin-15 gene, were selected for additional characterization. Nematodes with stable arrays ( $>50 \%$ transmittance) were analyzed by PCR for the presence of LRRK2 cDNA and chosen for chromosomal integrations. The LRRK2-1 primer set was used for the analysis (Table 1). L4 nematodes carrying arrays were subjected to $\gamma$-irradiation (3500-5000 rad) and grown further to generate F1 progenies. Five hundred F1 nematodes were individually grown in single plates and selected for $100 \%$ transmittance. With the yield of $2-3 \%$ stable integrants, transgenic nematodes were outcrossed with Bristol N2 nematodes (six times) and PCR analyzed for the presence of LRRK2 cDNA. The resulting integrated lines were labeled wlzIs1 and wlzIs2 for WT LRRK2 lines, wlzIs3 and wlzIs4 for the G2019S lines, wlzls5 for the R1441C line, wlzls6 for the KD line, and wlzls7 for the R1441C/KD line. LRRK2/DAT::GFP lines (carrying enhanced GFP) were created by conventional crossing of wlzIs2 and wlzIs4 with the DAT::GFP line and verified with single nematode PCR analysis.

Nematodes were synchronized by either bleaching method or letting nematodes lay eggs for $2-3 \mathrm{~h}$.

Brood size. This was determined by placing five to six single L4 nematodes onto individual plates and transferring the same nematode every day to a new plate until it stopped laying eggs. Laid eggs were counted for brood size determination.
Table 1. Amplimer sets used for PCR

\begin{tabular}{lll}
\hline Name & Forward sequence & Reverse sequence \\
\hline LRRK2-1 & 5'-ATGGCTAGTGGCAGCTGTCAG & 5'-GAGTCCAAGACGATCAACAGA \\
LRRK2-2 & 5'-AGCTTCCTCACGCAGTTCACTTT & 5'-TCACTTAACTGCAGTGCTGGGTCT \\
act-1 & 5'-CCCAATCCAAGAGAGGTATCCTT & 5'-AGGTGTGATGCCAGATCTTCTCCA \\
Irk-1 & 5'-GCAAGAGCATCAAGAGGAGAGAG & 5'-CGCAGAGGGTTTGAAGTGACAT \\
ben-1 & 5'-TCCAATGTTCGTGGTGGAGTCAA & 5'-TAGTGGTGCAGGAAGCATTGGAGA \\
\hline
\end{tabular}

Lifespan. Assays were performed without the conventional fluorodeoxyuridrne to avoid any complication from inhibition of DNA synthesis affecting the biology of LRRK2. Each point on the graph corresponded to results from three dishes of 40 nematodes each. Nematode viability was checked every day with a soft touch at the tip of pharynx of the nematode. Nematodes were transferred to fresh plates every other day.

Nematode viability. Assays were performed using three dishes of $30-40$ adult nematodes for each condition and strain. NGM agar contained specified concentrations of chemicals. For most studies, the concentrations of toxins was rotenone at $25 \mu \mathrm{M}$ and paraquat at $500 \mu \mathrm{M}$. Treatment with each toxin was begun on adult day 1 . Nematodes that crawled up the side of the plate or died as a result of egg laying defects were excluded from all analyses.

RNAi knockdown. This was performed according to Kamath et al. (2001). Ht115 bacteria was grown overnight in LB media containing 50 $\mu \mathrm{g} / \mathrm{ml}$ carbenecillin. Bacteria was then plated on NGM plates containing $1 \mathrm{~mm}$ isopropyl- $\beta$-D-thiogalactopyranoside (IPTG) and allowed to grow overnight at room temperature. Nematodes were synchronized by $3 \mathrm{~h}$ egg laying and exposed on adult day 1 to Ht115 bacteria containing the pL4440 expression vector with the lrk-1 open reading frame (ORF) (Open Biosystems) or empty vector. On adult day 3, 20 nematodes were moved in triplicate to plates containing $1 \mathrm{~mm}$ IPTG and rotenone (25 $\mu \mathrm{M})$, which were plated with lrk-1 ORF or empty vector containing bacteria. For RNAi validation nematodes were exposed to either empty vector or lrk-1 ORF containing bacteria for $4 \mathrm{~d}$, followed by analysis of gene expression. Analysis of the lrk-1 RNAi construct by the program dsCheck showed no significant off-target effects for the RNAi construct.

Quantitative real-time reverse transcription-PCR assay. Total RNA from C. elegans was extracted using Qiagen RNeasy mini kit. Intact RNA was checked by running a 1.0\% agarose/formaldehyde gel and quantified spectrometrically (NanoDrop ND-1000) before proceeding to subsequent steps. One milligram of total RNA was reverse transcribed using iScript cDNA synthesis kit (Bio-Rad) according to the instructions of the manufacturer instructions. Real-time reverse transcription (RT)-PCR was performed on a MyiQ Detection System (Bio-Rad) using iQ SYBR Green supermix. Expression levels for each target gene were calculated by the $2^{- \text {DDCT }}$ method (Livak and Schmittgen, 2001). All analyses were performed in triplicates. Primers used for the real-time RT-PCR are listed in Table 1. The amplimer sets used were LRRK2-2, act-1, lrk-1, and ben-1.

Dopamine measurements. The nematodes ( $\sim 1000$ per sample) were washed off $10 \mathrm{~cm}$ plates with water and then washed with M9 buffer until the adult nematodes were clean of bacteria. Nematode pellets $(30-40 \mu \mathrm{l})$ were measured and weighed. The samples were stabilized with $200 \mu \mathrm{l}$ of $0.1 \mathrm{~N}$ perchloric acid, vortexed, and then sonicated on ice in $10 \mathrm{~s}$ intervals and spun down at 14,000 rpm for $15 \mathrm{~min}$ to remove any insoluble residue. The samples were kept on ice, and the acid supernatant was decanted from the insoluble pellet. Forty to $60 \mu \mathrm{l}$ of the acid supernatant was transferred into an HPLC loading tube. The samples were injected into a Varian Microsorb C18 MV 100-5 (250 × 4-6 mm column). Eluted compounds were detected electrochemically using an ESA 542 HPLC autosampler.

Immunocapture. C. elegans were harvested, washed in M9 buffer three times to remove contaminating bacteria, frozen, pulverized, and dissolved in affinity binding buffer: radioimmunoprecipitation assay buffer plus protease inhibitors (HALT; Sigma). The worm lysates $(1 \mathrm{~g} / 3 \mathrm{ml})$ were sonicated, and the insoluble material was spun down. After passage through a $0.22 \mu \mathrm{m}$ filter, LRRK2 was captured from the supernatant using a column containing $1 \mathrm{ml}$ of anti-V5 agarose antibody. After $10 \mathrm{ml}$ 
A

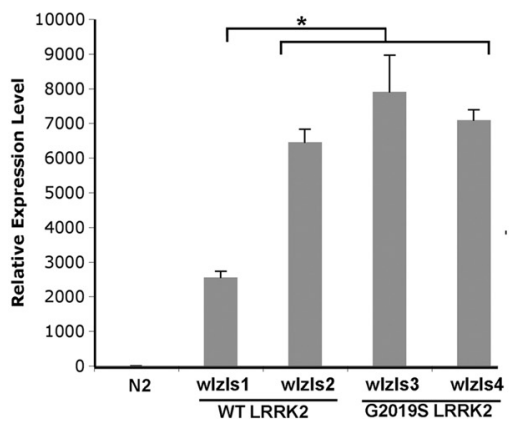

D

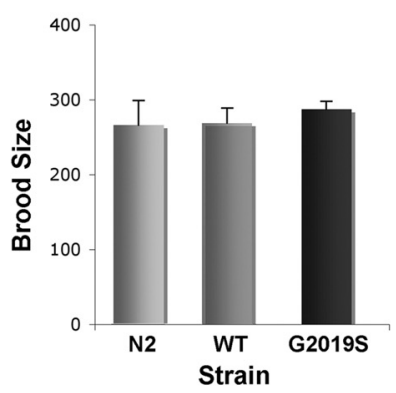

B

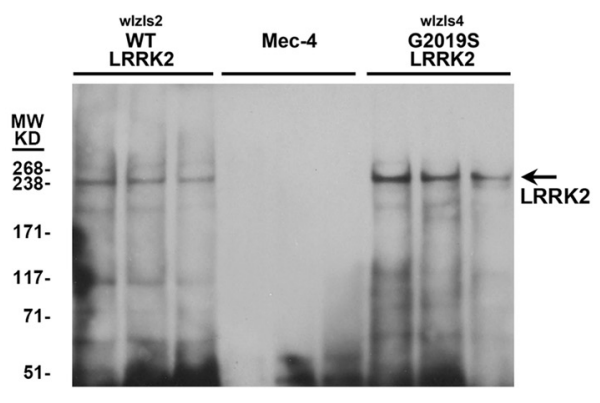

C

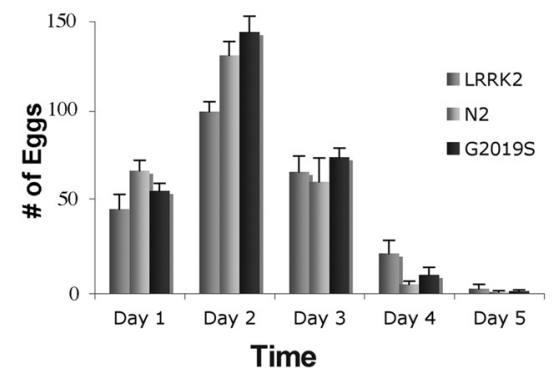

E

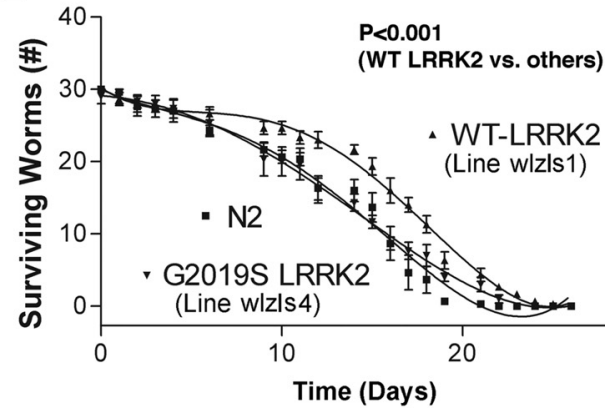

F

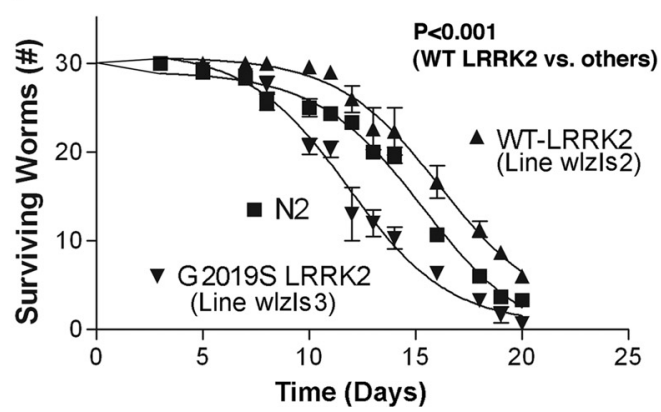

Figure 1. Generation and characterization of C. elegans lines expressing WT or G2019S LRRK2.A, Quantification of LRRK2 mRNA expression for the WT LRRK2 lines (wlzls1 and wlzls2) and G2019S LRRK2 lines (wlzls3 and wizls4). LRRK2 expression level was only significantly different for line wlzls $\left.1{ }^{*} p<0.001\right)$. B, Expression of LRRK2 in C. elegans lines. Three successive fractions eluted from the anti-V5 antibody column are shown for each nematode line. Lanes 1-3, WT LRRK2 (line wlzls2); lanes 4-6, Mec-4::GFP (a line derived from the same lin-15 background as the LRRK2 lines); lanes 7-9, G2019S LRRK2 (linewIzls4). The arrow points to the V5-LRRK2 at $250 \mathrm{kDa}$, which was eluted from the anti-V5 column. C, Number of eggs laid by N2 and transgenic lines. D, Analysis of total brood size for WT and G2019S LRRK2 (lines wizls2 and wlzls4) demonstrates similar levels of fertility. $E$, $F$, Analysis of lifespan for WT and G2019S LRRK2 shows that C. elegans expressing WT LRRK2 (lines wlzls1, wlzls2) exhibit greater median lifespan compared with nontransgenic (N2) or G2019S LRRK2 lines (lines wlzls3, wzls4). The lifespan data for $\boldsymbol{E}$ and $\boldsymbol{F}$ were generated with separate experiments.

of washing with PBS/0.1\% Tween 20/1 mm EDTA, material was eluted from the column with $1 \mathrm{mg} / \mathrm{ml} \mathrm{V5}$ peptide.

Immunoblotting. Sample was resuspended in Laemmli's buffer containing $1 \%$ SDS and $0.5 \% \beta$-mercaptoethanol and immediately heated at $95^{\circ} \mathrm{C}$ for $10 \mathrm{~min}$. Separation was done using 3-8\% NuPAGE Tris-acetate gels. Transfer was at $40 \mathrm{~V}$ for $75 \mathrm{~min}$. The immunoblot was blocked in $5 \%$ nonfat milk/0.05\% Tween 20/TBS. Antibodies used included the following: rabbit anti-V5 antibody (V8137; Sigma), anti-V5 agarose (V8137, 1:1000; Sigma), rabbit anti-LRRK2 (NB300-268; Novus), mouse anti-actin (mAb1501; Millipore Corporation), and peroxidase coupled donkey antimouse antibody (1:5000; Jackson ImmunoResearch). Development was with enhanced chemiluminescence (Pierce).

Optical analysis of GFP fluorescence. Synchronized adult nematodes were treated as described above. GFP fluorescence was measured 1-2 d after each treatment, using Carl Zeiss Axiovision software for the analyses. For quantification, rectangles were placed around the CEP dopaminergic neurons (cell bodies), which are the most prominent neurons, and the mean optical density was determined; the area between the neurons was used to define background optical density.

Statistical analysis. Results are presented as mean \pm SEM with ANOVA for analysis and Newman-Keuls post hoc testing. Lifespan was analyzed by hazard rate analysis using GraphPad Prism software.

\section{Results}

\section{Characterization of LRRK2 C. elegans strains}

LRRK2 was expressed in C. elegans to elucidate its in vivo functions and to understand how mutations affect LRRK2 function. WT or G2019S LRRK2 were inserted into the pSybDEST plasmid. This plasmid contains a synaptobrevin (SNB) promoter, which drives neuronal-selective expression of LRRK2. The lines were selected with a lin-15 coinjection marker plasmid by injecting into a temperature-sensitive lin-15 strain (n765ts) (Clark et al., 1994). The presence of WT and mutant LRRK2 genes was con- firmed by PCR. The WT and G2019S LRRK2 lines were subsequently integrated with $\gamma$-irradiation. Two independent lines for each transgene were selected, and each line was subsequently outcrossed six times with nontransgenic Bristol N2 to remove the lin-15 background and unwanted mutations from the integration process. Expression of LRRK2 in the resulting lines (WT LRRK2, lines wlzIs1 and wlzIs2, and G2019S LRRK2, lines wlzIs3 and wlzIs4) was quantified by real-time RT-PCR (Fig. 1A). The expression levels among lines wlzIs2, wlzIs3, and wlzIs4 were comparable, whereas line wlzIs1 showed somewhat lower LRRK2 expression (Fig. 1A). We also performed immunoblots to verify protein expression. Nematode lysates were generated and V5LRRK2 was immunocaptured on an anti-V5 antibody column, eluted with V5 peptide, and immunoblotted with anti-V5 antibody. A single band migrating at $250 \mathrm{kDa}$ was present in the WT and G2019S LRRK2 samples corresponding to the V5 peptide eluate (Fig. $1 \mathrm{~B}$ ). Although immunocapture is not quantitative, expression of LRRK2 appeared to be higher in the G2019S LRRK2 line (wlzls4) than the WT line (wlzls2) (Fig. $1 B$ ). V5-LRRK2 was absent from the Mec-4::GFP, which was used as a negative control. The Mec-4::GFP line was used as a control for the LRRK2 lines because this line was derived from the same temperaturesensitive lin-15 strain (n765ts) as the LRRK2 lines (Clark et al., 1994; Xu et al., 2001). LRRK2 expression was also validated by the ability of WT and G2019S LRRK2 to complement a phenotype (enhanced sensitivity to rotenone) associated with knockdown or partial deletion of C. elegans lrk-1 (see Fig. 3C).

Each of the LRRK2 nematode lines was able to develop through the larval stage and into adulthood without any gross deformities. Small differences in the pattern of egg production in 
A

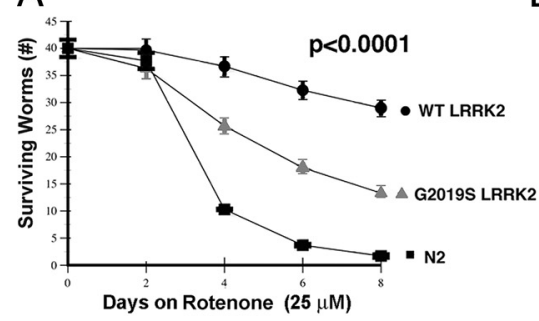

B
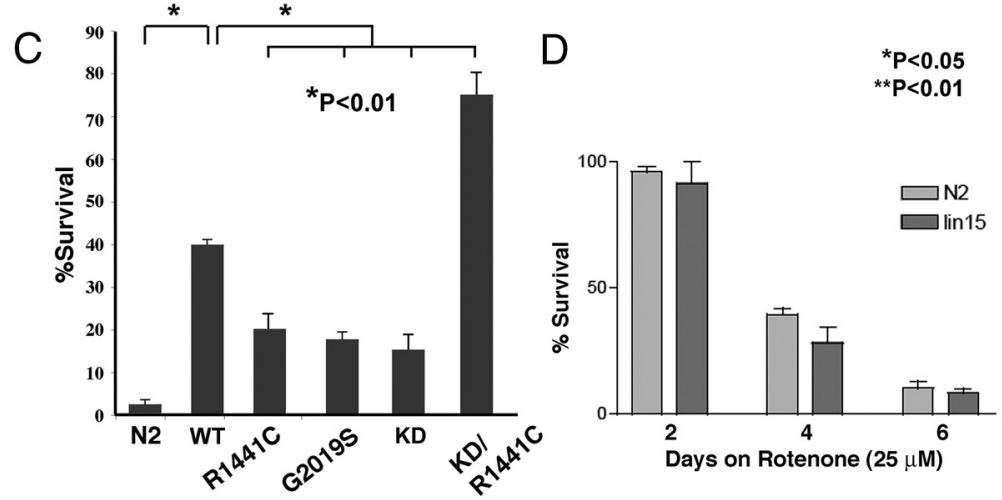

Figure 2. LRRK2 protects against mitochondrial stress. $A$, Nematode expressing WT or G2019S LRRK2 (lines wlzls2 and wIzls4) showed reduced toxicity to rotenone $(25 \mu \mathrm{M})$. $\boldsymbol{B}$, Nematodes expressing WT LRRK2 (strain wlzls2) showed reduced toxicity to paraquat $(500 \mu \mathrm{M}$ ). The G2019S LRRK2 strain (strain wlzls4) showed sensitivity to paraquat similar to that of N2. The $p$ values represent overall $p$ values for each figure. WT and G2019S LRRK2 were significantly different $(p<0.0001)$ in $\boldsymbol{A}$ and $\boldsymbol{B} ; \mathrm{N2}$ and G2019S LRRK2 were not significantly different in B. C, R1441C, G2019S, and KD LRRK2 (wlzls5, wlzls4, and wlzls7) showed less protection against rotenone (10 $\mu \mathrm{M}$ ) than WT LRRKS (wIzls2), and KD/R1441C LRRK2 (wlzls6) was strongly protected against rotenone $(20 \mu \mathrm{m}) \mathrm{N} 2$ ( $n=12,40$ nematodes per dish for all points). $\boldsymbol{D}$, Bristol $\mathrm{N} 2$ and lin-15 (the background strain for used for producing the transgenic $C$. elegans lines) showed similar levels of sensitivity to rotenone ( $25 \mu \mathrm{M}, 4 \mathrm{~d}$ ). the R1441C and KD mutations. The R1441C (wlzls5) and KD (wlzls6) LRRK2 nematode lines showed little protection against rotenone (Fig. 2C). Surprisingly, the KD/R1441C line (wlzls7) showed striking protection against rotenone toxicity (Fig. 2C). LRRK2 protein levels in each line were assessed by V5 immunocapture, followed by immunoblotting with anti-V5 antibody (supplemental Fig. 1, available at www.jneurosci.org as supplemental material).

We also examined whether the increased sensitivity of the LRRK2 lines could be attributed to expression of the lin-15 transgene, which was used for selecting transgenic lines by complementation of the parent line lin-15 (n765ts) (Clark et al., 1994). To test the effects of the lin-15 transgenesis method, we used a nematode line expressing GFP driven by the Mec-4 promoter (SK4005), which was selected by lin-15 complementation ( $\mathrm{Su}$ zuki et al., 2003). The differences in rotenone sensitivity were not attributable to complementation because Bristol N2 exhibited a sensitivity to rotenone similar to that of Mec-4::GFP line (Fig. 2D, $6 \mathrm{~d}$ time course). We also examined the ST2 line of C. elegans, which has widespread neuronal GFP expression to examine whether panneuronal expression of a protein unrelated early adulthood were evident, but these differences did not reach statistical significance (Fig. $1 C$ ), and brood sizes were similar between the WT and G2019S nematodes (Fig. 1D). Analysis of lifespan in age-synchronized populations of nematodes showed an effect of LRRK2 on longevity. WT LRRK2 lines exhibited increased median lifespans compared with G2019S LRRK2 and N2 strains (Fig. $1 E, F)(p<0.0001)$.

\section{LRRK2 reduces vulnerability to mitochondrial dysfunction}

PD-related genetic modifications frequently render neurons more vulnerable to mitochondrial complex I inhibition (Betarbet et al., 2000; Dauer and Przedborski, 2003; Ved et al., 2005). We therefore examined whether LRRK2 expression modified the response to mitochondrial inhibition in C. elegans. Age-synchronized lines of young adult WT and G2019S LRRK2 C. elegans were exposed to $25 \mu \mathrm{M}$ rotenone beginning adult day 1 , and survival was measured every $2 \mathrm{~d}$ for $8 \mathrm{~d}$. After $8 \mathrm{~d}$ of treatment, survival was $80 \%$ for WT LRRK2, 40\% for G2019S LRRK2, and 2\% for nontransgenic (Fig. 2A). To understand whether LRRK2 protected against other forms of mitochondrial inhibition, we investigated vulnerability to the mitochondrial toxin paraquat. N2 C. elegans were sensitive to $500 \mu \mathrm{M}$ paraquat and exhibited $4 \pm 2 \%$ survival at $14 \mathrm{~d}$ (Fig. 2 B). C. elegans expressing WT LRRK2 were much more resistant to paraquat and exhibited $85 \pm 4 \%$ survival at $14 \mathrm{~d}$ (Fig. 2B). G2019S LRRK2 did not protect $C$. elegans against paraquat and gave a profile similar to that of $\mathrm{N} 2$ C. elegans (Fig. $2 B$ ). Survival of the WT LRRK2 line was more pronounced than could be accounted for by differences in baseline viability at that age (Fig. 1E). We also examined the effects of other LRRK2 mutations on sensitivity to rotenone, including the R1441C and KD LRRK2 mutations, as well as a LRRK2 construct containing both to LRRK2 affected the vulnerability to rotenone (Shioi et al., 2001). We observed no difference in vulnerability to rotenone (supplemental Fig. 2, available at www.jneurosci.org as supplemental material).

\section{Endogenous C. elegans lrk-1 also modulates the response to mitochondrial dysfunction}

Knockdown of endogenous lrk-1 had effects opposite to expressing LRRK2. Bristol N2 nematodes were fed bacteria containing an RNAi expression plasmid for lrk-1 to test whether endogenous lrk-1 activity modulates sensitivity to mitochondrial inhibition. Two days after feeding was initiated, nematodes were treated with rotenone $(25 \mu \mathrm{M})$ and assayed over the next $4 \mathrm{~d}$. Knockdown of lrk-1 did not affect baseline viability (100\% survival at day 4$)$ but did sensitize $C$. elegans against mitochondrial inhibition compared with knockdown of either empty vector or sel-9 (Fig. 3A, empty vector comparison shown). Knockdown of lrk-1 was verified by real-time RT-PCR. Levels of lrk-1 were $46 \pm 5 \%(p<$ 0.05 ) lower in nematodes exposed to lrk-1 RNAi compared with nematodes exposed to the PL4440 empty RNAi vector. The reciprocal effects of lrk-1 knockdown and LRRK2 expression support a hypothesis that the effects of LRRK2 expression in C. elegans derive from actions linked to the endogenous function of lrk-1.

Next, we proceeded to examine the effects of partial lrk-1 deletion to test the knockdown results through an independent mechanism. The lrk-1 [km17] C. elegans strain has a deletion eliminating the kinase and WD40 domains of lrk-1, the C. elegans ortholog of LRRK2 (Sakaguchi-Nakashima et al., 2007). We used the lrk-1 [km17] strain to examine the effects of deleting the lrk-1 kinase domain on the response to mitochondrial inhibition. Adult lrk-1 [km17] nematodes (adult, day 1) were treated with rotenone $(25 \mu \mathrm{M})$ for $4 \mathrm{~d}$. The lrk-1 [km17] strain showed en- 

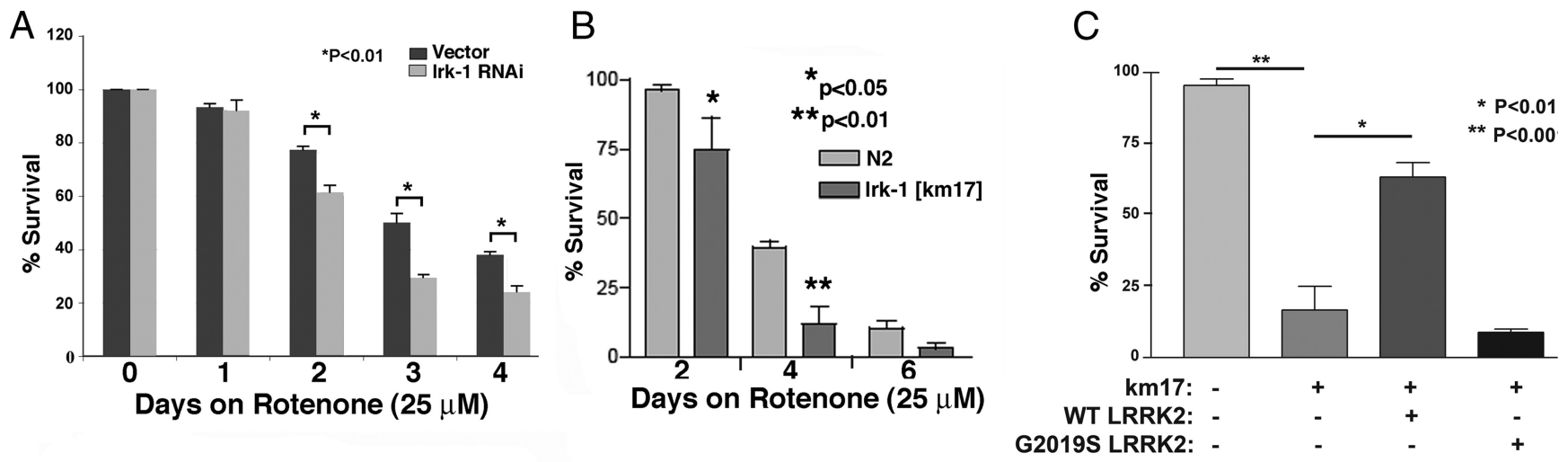

Figure 3. Endogenous C. elegans Irk-1 modulates the response to mitochondrial stress. $A$, Knockdown of Irk-1 enhanced rotenone ( $25 \mu \mathrm{M})$ toxicity of Bristol N2 C. elegans. $\boldsymbol{B}$, The Irk-1 [km17] line, lacking the Irk-1 kinase and WD40 domains, showed reduced protection against rotenone compared with the control Bristol N2 line. C, WT LRRK2 (wlzsls2), but not G2019S (wlzsIs4), partially complemented the Irk-1 [km17] deletion after $4 \mathrm{~d}$ on $10 \mu \mathrm{m}$ rotenone (data normalized to the parent line).

hanced vulnerability to rotenone (Fig. 3B). The km17 deletion exerted no affect on baseline viability $C$. elegans over the $4 \mathrm{~d}$ time span used for the experiment (supplemental Fig. 3, available at www.jneurosci.org as supplemental material).

We also examined whether LRRK2 expression complemented the lrk-1 [km17] phenotypes. The SNB::GFP/lrk-1 [km17] line was crossed onto the WT and G2019S LRRK2 backgrounds, and the sensitivity to rotenone $(10 \mu \mathrm{M}, 4 \mathrm{~d})$ was measured (Sakaguchi-Nakashima et al., 2007). Protection against rotenone toxicity was observed for the SNB::GFP/lrk-1 [km17]/WT LRRK2 line but not for the SNB::GFP/lrk-1 [km17]/G2019S LRRK2 (Fig. 3C). The km17 deletion exerted no effect on baseline viability of C. elegans over the $4 \mathrm{~d}$ time span used for the experiment, although after day 5, the line carrying the lrk-1 [km17] mutation began to show more toxicity than the parent line juls137. Neither the SNB::GFP nor the km17 deletion exerted an affect on baseline viability C. elegans over the $4 \mathrm{~d}$ time span used for the experiment (supplemental Fig. 4, available at www. jneurosci.org as supplemental material). WT LRRK2 also complemented the enhanced rotenone sensitivity observed with knockdown of lrk-1. RNAi for lrk-1 and pL4440 were associated with 25 and $75 \%$ toxicity $(p<0.01,25 \mu \mathrm{M}$ rotenone, $2 \mathrm{~d}$ ), respectively. Complementation by WT LRRK2 in the rotenone assay supports the hypothesis that LRRK2 complements the loss of function present in the lrk-1 [km17] strain.

Using the same lines, we examined whether LRRK2 complemented an SNB mislocalization phenotype putatively associated with the lrk-1 [km17] strain (Sakaguchi-Nakashima et al., 2007). SNB does not normally reside in the dendrites, but in the lrk-1 [km17] strain, SNB expression is present at the dendritic tip of the amphid sensory neuron. Surprisingly, neither WT LRRK2 nor G2019S LRRK2 modified SNB::GFP fluorescence in the distal amphid (supplemental Fig. 5, available at www.jneurosci.org as supplemental material). Thus, expressing WT LRRK2 modulated SNB::GFP expression but did not complement the mislocalization observed in the SNB::GFP/lrk-1 [km17] line.

\section{Dopaminergic marker loss is greater in C. elegans expressing G2019S LRRK2 than WT LRRK2}

Dopaminergic neurons show selective vulnerability in subjects with LRRK2 mutations. We used the ability to track individual neurons in C. elegans in vivo to examine the effects of LRRK2 on dopaminergic neurons. WT and G2019S LRRK2 lines (lines wlzIs2 and wlzIs4) were crossed with a C. elegans line expressing
GFP driven by the dopamine transporter-1 (DAT) promoter DAT::GFP (strain 410), and the DAT::GFP fluorescence was monitored (Kuwahara et al., 2006). Previous work by Nass et al. (2002) demonstrated that loss of fluorescence of a DAT::GFP construct is associated with degeneration of dopaminergic neurons in C. elegans. Age-synchronized C. elegans lines expressing LRRK2 (WT and G2019S) showed strong dopaminergic GFP signal during the $\mathrm{L} 3$ stage that was similar to or slightly stronger than the 410 control line (Fig. $4 A, B$ ). On reaching adulthood, the dopaminergic signal showed a rapid decrease in LRRK2 lines, with the G2019S LRRK2/DAT::GFP line showing a significantly greater decrease in signal. By day 2 of adult life, the signal of G2019S LRRK2/DAT::GFP line was 80\% less than the control 410 DAT::GFP line and 60\% less than the WT line (Fig. 4A-C). Additional aging produced loss of GFP signal such that, by day 5 , fluorescence was reduced to similar levels in all the nematode lines.

Loss of dopaminergic markers was confirmed by measuring dopamine levels. Synchronized lines of DAT::GFP and WT or G2019S LRRK2/DAT::GFP C. elegans lines were harvested at adult day 3 , and levels of dopamine were measured by HPLC. Dopamine levels in the DAT::GFP and WT LRRK2/DAT::GFP lines were not significantly different, although there was a trend toward a decrease in dopamine in the WT LRRK2 line (Fig. 4D). This contrasts with the increased loss of DAT::GFP fluorescence observed in the WT LRRK2/DAT::GFP line and indicates that, under some conditions, DAT::GFP fluorescence and dopamine levels are not linked. The G2019S LRRK2/DAT::GFP line showed significantly less dopamine than WT LRRK2/DAT::GFP or DAT::GFP lines (Fig. $4 D$ ). These results support the hypothesis that C. elegans expressing G2019S LRRK2 show enhanced loss of dopaminergic neurons.

The effects of LRRK2 on GFP fluorescence in a different set of neurons, those expressing Mec-4, were also examined to assess whether LRRK2 exerts effects that are selective for dopaminergic neurons (supplemental Fig. 6, available at www.jneurosci.org as supplemental material) (Xu et al., 2001). Mec-4 is present in neurons sensitive to touch, including a pair in the tail (Xu et al., 2001). C. elegans expressing Mec-4::GFP were crossed with WT LRRK2 (line wlzIs2) or G2019S LRRK2 (line wlzIs4). Fluorescence of the Mec-4::GFP construct was assessed in each nematode line from birth up to adult day 6 (supplemental Fig. 6, available at www.jneurosci.org as supplemental material). Mec-4::GFP fluorescence was present in each of the nematode lines, and fluorescence intensity was not altered by the presence of WT or G2019S 


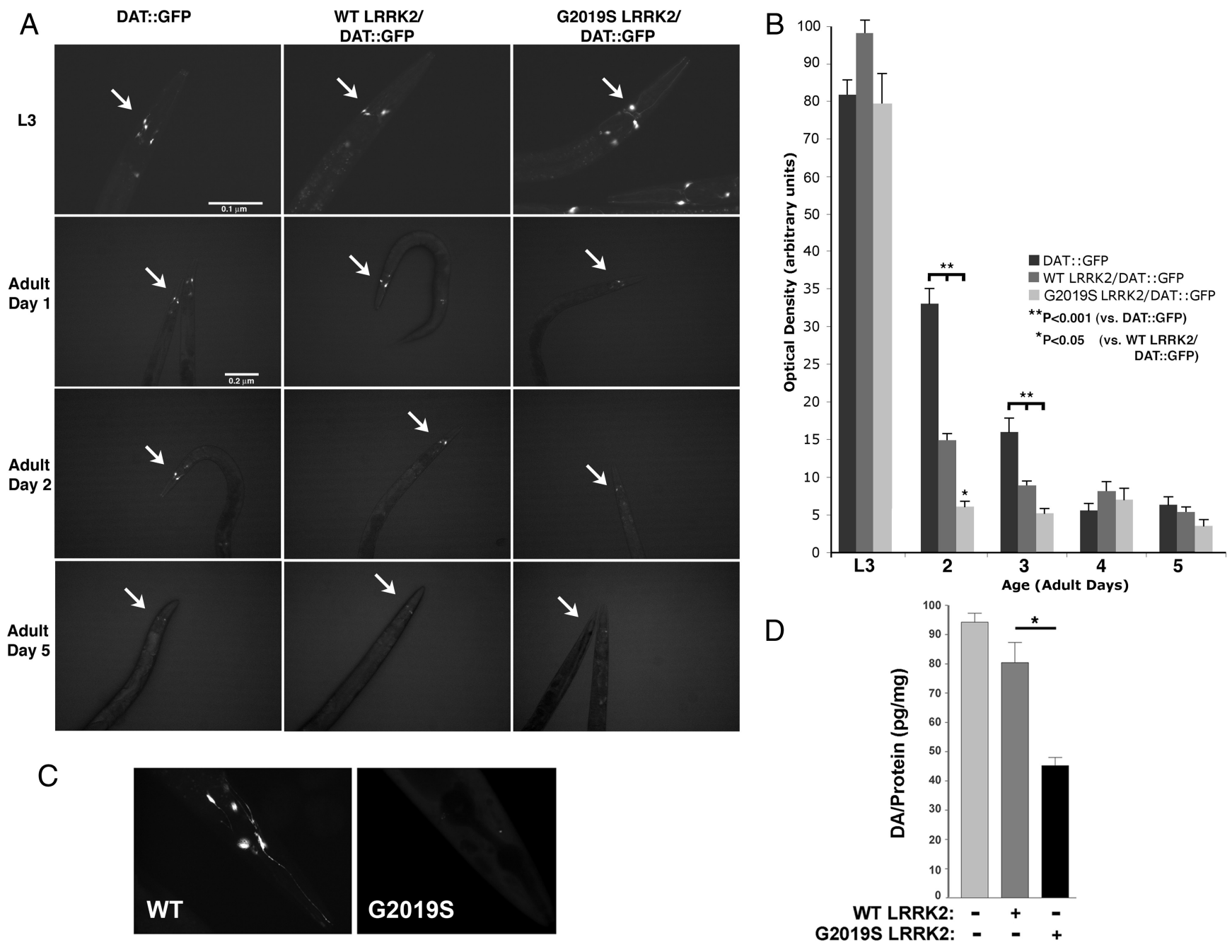

Figure 4. LRRK2 induces loss of DAT::GFP fluorescence in dopaminergic neurons of adult nematodes. A, DAT::GFP fluorescence at different ages. The arrows point to the CEP dopaminergic neurons. The LRRK2 lines showed an accelerated decrease in DAT::GFP signal. $B$, Quantification of fluorescence signal from the nematode lines beginning with larval stage 3 and continuing on in days of adult life. C, High magnification (100X) showing representative DAT::GFP fluorescence in the WT and G2019S LRRK2 lines at day 2. D, G2019S LRRK2 (line wlzls4) shows reduced dopamine levels at day 2 of adult life compared with the WT LRRK2 (line wlzls2) or nontransgenic (N2) line $\left.{ }^{*} p<0.05\right)$.

LRRK2 at any time point as judged by either visual or quantitative methods (supplemental Fig. 6, available at www.jneurosci.org as supplemental material). These data suggest that G2019S LRRK2 stimulates loss of dopaminergic markers in C. elegans in a manner that is particularly evident early in the adult phase of the nematode life cycle and that the changes observed exhibit at least partial selectivity for dopaminergic neurons.

\section{G2019S LRRK2 sensitizes dopaminergic neurons to} mitochondrial inhibition compared with WT LRRK2

We proceeded to examine interactions between LRRK2 and toxin exposure on dopaminergic neurons. We examined the effects of LRRK2 on the response of dopaminergic neurons to rotenone. DAT::GFP fluorescence was highly sensitive to rotenone, necessitating use of a lower dose $(250 \mathrm{~nm})$ of rotenone than for survival assays. Dopaminergic neurons in WT LRRK2/DAT::GFP retained strong fluorescence after exposure to $250 \mathrm{nM}$ rotenone for $24 \mathrm{~h}$, whereas dopaminergic neurons in the control DAT::GFP or G2019S LRRK2/DAT::GFP lines showed $55 \pm 16$ and $83 \pm 5 \%$ loss of fluorescence, respectively (Fig. 5A, B). Dopamine levels in the WT LRRK2/DAT::GFP lines treated with rotenone (250 nM) showed enhanced protection compared with the Bristol N2 line under the same conditions, which parallels the fluorescence data (Fig. 5C). The G2019S LRRK2 line showed dopamine levels that were significantly less than that of the WT LRRK2 line after rotenone treatment but similar to that of the N2 line. These data suggest that the G2019S LRRK2 construct does not provide the same level of protection as the WT LRRK2 construct.

We also examined the response of lrk-1 [km17] and Mec-4 neurons to rotenone treatment. To examine the lrk-1 [km17] line, nematodes expressing DAT::GFP were crossed to lrk-1 [km17]. The lrk-1 [km17]/DAT::GFP and DAT::GFP lines were then age synchronized and treated with or without rotenone $(250 \mathrm{nM}, 24 \mathrm{~h})$ beginning at adult day 1 . Optical densities for the lrk-1 [km17]/DAT::GFP line $(n=8-10)$ were normalized to the DAT::GFP line under the same conditions. The lrk-1 [km17]/DAT::GFP showed enhanced loss of DAT::GFP fluorescence in response to rotenone treatment compared with control ( $32 \pm 9, p<0.01, n=10,250 \mathrm{nM}, 24 \mathrm{~h}$ ). This indicates that loss of lrk-1 function has the opposite effect from overexpressing WT LRRK2. Parental, WT and G2019S LRRK2/Mec-4::GFP lines were age synchronized, and young adult nematodes were exposed to rotenone ( $250 \mathrm{~nm}, 3 \mathrm{~d}$ ). No differences in GFP fluorescence were observed between the 
Mec-4::GFP, Mec-4::GFP/WT LRRK2, and Mec-4::GFP/G2019S LRRK2 lines (data not shown).

\section{Discussion}

Using C. elegans as a model, we observed that dopaminergic neurons were particularly sensitive to mutant LRRK2. G2019S LRRK2 increased vulnerability of dopaminergic neurons relative to WT LRRK2 lines both basally and in response to mitochondrial stress. Survival in the nematode survival assay was also decreased for mutant LRRK2 (G2019S or R1441C) relative to WT LRRK2, but all constructs increased survival relative to controls (nontransgenic N2 or Mec-4). The contrasting responses of the dopaminergic neuron assay compared with the survival assay might reflect responses of different types of cells because dopaminergic neurons are not required for survival. Knockdown as well as deletion of lrk-1 reduced nematode survival in response to rotenone treatment, which is opposite to the response to LRRK2 expression, and suggests that the survival phenotype can be attributed to endogenous functions of lrk-1.

Our results indicate a role for LRRK2 and its $C$. elegans ortholog lrk-1 in regulation of mitochondrial dysfunction. Rotenone and paraquat act by inhibiting the mitochondrial electron transport chain, which leads to a number of effects, including increased free radical production and reduced ATP (Yamada and Fukushima, 1993; Castello et al., 2007). Rotenone and paraquat act at different sites on the respiratory chain (Fukushima et al., 1993, 1994). Rotenone and paraquat also have pleiotropic actions, which include interfering with microtubule function and activating transcription factors ( $\mathrm{Li}$ and Sun, 1999; Ren et al., 2003, 2009). It is possible that the differences in protection in response to rotenone and paraquat in nematodes expressing G2019S LRRK2 might reflect actions beyond the mitochondria. Oxidative stress and reduced energy production lead to many other cellular effects, but these changes are grouped under a broad term, mitochondrial dysfunction, which is meant to encompass these effects. We also observed that G2019S LRRK2 enhances the loss of DAT::GFP fluorescence in adult dopaminergic neurons in response to mitochondrial dysfunction (compared with WT LRRK2). This process appears to be at least partially selective for dopaminergic neurons, because LRRK2 did not affect the vulnerability of Mec- 4 neurons, as defined by fluorescence intensity of an Mec-4::GFP construct.

\section{LRRK2 and lrk-1 appear to have similar functions in C. elegans}

The studies in C. elegans shed light on the biology of LRRK2 and lrk-1. C. elegans express only one LRRK, lrk-1, whereas mammals express LRRK1 and LRRK2. The lrk-1 protein exhibits strong regions of homology to human LRRK1 and LRRK2. Homology searches indicate that the lrk-1 protein has $24 / 40 \%$ homology (exact/ positives matches) to LRRK1 over a region of $\sim 1500$ aa and $25 / 44 \%$ homology (exact/positive matches) to LRRK2 over a region of $\sim 1300$ aa.
Our results indicate a role for LRRK2 and lrk-1 in regulating cellular responses to stress. The putative role of LRRK2 as a stress response protein is supported by reciprocal actions associated with LRRK2 expression and lrk-1 knockdown or deletion. RNAi can have off-target effects, but off-target effects are unlikely to be a major factor modifying our results because we used two different RNAi controls. In addition, deletion of the lrk-1 kinase and WD40 domains in C. elegans lrk-1 produces sensitization to rotenone similar to that observed with lrk-1 knockdown. The similar effects observed for lrk-1 RNAi and the lrk-1 [km17] deletion strain after rotenone treatment suggest that the lrk-1 RNAi was active, although some RNAi are not effective in neurons so we cannot rule out changes in survival mediated by humoral factors. The ability of LRRK2 to complement the rotenone sensitization associated with the lrk-1 [km17] strain provide additional support for the hypothesis that LRRK2 and lrk-1 share similar functions.

An increasing number of studies suggest a role for LRRK2 in regulating neuronal responses to stress. Transient expression of LRRK2, but not LRRK1, is toxic to neurons (Greggio et al., 2007); PD-linked mutations in LRRK2 show enhanced toxicity (West et al., 2005, 2007; Greggio et al., 2006; MacLeod et al., 2006; Smith et al., 2006). In Drosophila, knockdown of LRRK2 enhances toxicity to rotenone (Lee et al., 2007). A putative role for LRRK2 in regulating neuronal responses to mitochondrial dysfunction is also supported by recent studies showing that LRRK2 associates with the outer mitochondrial membrane (Biskup et al., 2006). Combining our results with those in the literature suggests a consistent line of evidence implicating LRRK2 with mitochondrial function.

We also examined complementation by examining the expression of synaptobrevin in the lrk-1 [km17] strain but did not observe an effect. The result could reflect two possibilities. First, 
WT LRRK2 might not complement the lrk-1 [km17] SNB mislocalization phenotype. Second, the SNB::GFP mislocalization in SNB::GFP/lrk-1 [km17] strain might not be a lrk-1-specific phenotype but might rather originate from a cosegregating gene mutation. Additional research will clarify this issue.

\section{Studies of disease-related mutations in LRRK2}

We examined the actions of two common disease-related LRRK2 mutations, G2019S and R1441C, as well as a LRRK2 construct containing three mutations that inactivate the kinase domain (Greggio et al., 2006). Each of these constructs showed less protection against mitochondrial inhibition than WT LRRK2 and rendered neurons more vulnerable to toxicity than WT LRRK2, which is consistent with previous reports in the literature (Greggio et al., 2006; Smith et al., 2006; West et al., 2007). However, both mutant strains still retain some protective activity and are moderately protected against rotenone compared with the control Bristol N2 strain. Interpretation of differences among the LRRK2 strains must be tempered by the experimental limitations of our study. Semiquantitative measurements by immunoblot, based on immunocapture from an anti-V5 column, suggest differences in the levels of LRRK2 protein expression among the different lines. The rank order of protein expression appears to be $\mathrm{G} 2019 \mathrm{~S}>\mathrm{WT}=\mathrm{R} 1441 \mathrm{C}>\mathrm{R} 1441 \mathrm{C} / \mathrm{KD}>\mathrm{KD}$ (Fig. 1 B). Vulnerability to rotenone among the lines correlates best with presence of a LRRK2 mutation; there is no clear correlation with LRRK2 protein expression level. For instance, the KD/R1441C line shows strong protection despite low-level expression. The strong protection against rotenone afforded by the KD/R1441C LRRK 2 construct contrasts with vulnerability of the KD line and suggests that LRRK2 kinase activity is not required for protection against rotenone toxicity in C. elegans but also points to the complex relationship between mutations and activity. The presence of two mutations in LRRK2 compensate for the loss of function of each particular mutation; we hypothesize that this might reflect dynamic interplay between different LRRK2 domains that alters the three-dimensional organization of the LRRK2 protein. The modest protection by the KD LRRK2 construct contrasted with enhanced rotenone sensitivity by the lrk-1 [km17] line. The simplest explanation is that the lrk-1 [km17] mutation has reduced lrk-1 levels, for instance, attributable to generating a truncated protein that is rapidly degraded. Alternatively, the kinase and WD40 domains might have binding functions that are separate from the kinase function but are required for protection against rotenone. The strong protection afforded by the KD/R1441C construct is striking and presents an opportunity for additional exploration into the structure/function relationships of LRRK2.

\section{LRRK2 modifies the response of dopaminergic neurons to mitochondrial dysfunction}

One of the more intriguing aspects of our study is the sensitivity of dopaminergic neurons to expression of LRRK2. The changes in vulnerability induced by LRRK2 in C. elegans dopaminergic neurons were more selective than the changes in vulnerability observed with the whole worm survival assay. Both WT and G2019S LRRK2 enhanced the vulnerability of dopaminergic neurons under basal growth conditions. WT LRRK2 protected dopaminergic neurons against rotenone toxicity. In contrast, G2019S LRRK2 renders dopaminergic neurons more vulnerable to rotenone treatment compared with WT LRRK2 lines, although the G2019S LRRK2 did retain some protective action relative to the control DAT::GFP line.

The molecular mechanisms by which LRRK2 protects against mitochondrial dysfunction remain to be determined. The data in this manuscript provide support for a hypothesis that LRRK2 might act in part by regulating mitochondrial function. Genetic and environmental evidence point to a role for mitochondrial dysfunction in PD. PINK1 localizes to the mitochondria and associates with mitochondrial proteins, such as HtrA2 (Valente et al., 2004; Plun-Favreau et al., 2007). Several PD genes, including PINK1, parkin, DJ-1, HtrA2, and $\alpha$-synuclein, modify mitochondrial function and might act in similar pathways (Moore et al., 2005; Ved et al., 2005; Yang et al., 2006; Exner et al., 2007). A recent microarray study implicates LRRK2 with protein pathways mediated by p53 and small GTPases (Häbig et al., 2008). Other studies indicate the LRRK2 interacts with hsp-90 and 4E-BP (Imai et al., 2008). GTPases were also identified as important modifiers of $\alpha$-synuclein toxicity (Cooper et al., 2006; Gitler et al., 2008). LRRK2 might act through any of the signaling pathways that regulate stress responses. Understanding integration of these signaling processes is particularly relevant to the biology of dopaminergic neurons, in which the interplay of multiple factors appears to account for the strong sensitivity to stresses linked to PD (Mosharov et al., 2009). Elucidating how LRRK2 interacts with these biochemical pathways could illuminate how LRRK2 contributes to the pathophysiology of PD.

Note added in proof. While this manuscript was in press, Li et al. (2009) published on mice overexpressing R1441G LRRK2, and also observed dopaminergic degeneration.

\section{References}

Betarbet R, Sherer TB, MacKenzie G, Garcia-Osuna M, Panov AV, Greenamyre JT (2000) Chronic systemic pesticide exposure reproduces features of Parkinson's disease. Nat Neurosci 3:1301-1306.

Biskup S, Moore DJ, Celsi F, Higashi S, West AB, Andrabi SA, Kurkinen K, Yu SW, Savitt JM, Waldvogel HJ, Faull RL, Emson PC, Torp R, Ottersen OP, Dawson TM, Dawson VL (2006) Localization of LRRK2 to membranous and vesicular structures in mammalian brain. Ann Neurol 60:557-569.

Brenner S (1974) The genetics of Caenorhabditis elegans. Genetics 77:71-94. Castello PR, Drechsel DA, Patel M (2007) Mitochondria are a major source of paraquat-induced reactive oxygen species production in the brain. J Biol Chem 282:14186-14193.

Chandra S, Fornai F, Kwon HB, Yazdani U, Atasoy D, Liu X, Hammer RE, Battaglia G, German DC, Castillo PE, Südhof TC (2004) Doubleknockout mice for alpha- and beta-synucleins: effect on synaptic functions. Proc Natl Acad Sci U S A 101:14966-14971.

Clark SG, Lu X, Horvitz HR (1994) The Caenorhabditis elegans locus lin-15, a negative regulator of a tyrosine kinase signaling pathway, encodes two different proteins. Genetics 137:987-997.

Cooper AA, Gitler AD, Cashikar A, Haynes CM, Hill KJ, Bhullar B, Liu K, Xu K, Strathearn KE, Liu F, Cao S, Caldwell KA, Caldwell GA, Marsischky G, Kolodner RD, Labaer J, Rochet JC, Bonini NM, Lindquist S (2006) Alpha-synuclein blocks ER-Golgi traffic and Rab1 rescues neuron loss in Parkinson's models. Science 313:324-328.

Dauer W, Przedborski S (2003) Parkinson's disease: mechanisms and models. Neuron 39:889-909.

Exner N, Treske B, Paquet D, Holmström K, Schiesling C, Gispert S, CarballoCarbajal I, Berg D, Hoepken HH, Gasser T, Krüger R, Winklhofer KF, Vogel F, Reichert AS, Auburger G, Kahle PJ, Schmid B, Haass C (2007) Loss-of-function of human PINK1 results in mitochondrial pathology and can be rescued by parkin. J Neurosci 27:12413-12418.

Fukushima T, Yamada K, Isobe A, Shiwaku K, Yamane Y (1993) Mechanism of cytotoxicity of paraquat. I. NADH oxidation and paraquat radical formation via complex I. Exp Toxicol Pathol 45:345-349.

Fukushima T, Yamada K, Hojo N, Isobe A, Shiwaku K, Yamane Y (1994) Mechanism of cytotoxicity of paraquat. III. The effects of acute paraquat exposure on the electron transport system in rat mitochondria. Exp Toxicol Pathol 46:437-441.

Gitler AD, Bevis BJ, Shorter J, Strathearn KE, Hamamichi S, Su LJ, Caldwell KA, Caldwell GA, Rochet JC, McCaffery JM, Barlowe C, Lindquist S 
(2008) The Parkinson's disease protein alpha-synuclein disrupts cellular Rab homeostasis. Proc Natl Acad Sci U S A 105:145-150.

Greene JC, Whitworth AJ, Kuo I, Andrews LA, Feany MB, Pallanck LJ (2003) Mitochondrial pathology and apoptotic muscle degeneration in Drosophila parkin mutants. Proc Natl Acad Sci U S A 100:4078-4083.

Greggio E, Jain S, Kingsbury A, Bandopadhyay R, Lewis P, Kaganovich A, van der Brug MP, Beilina A, Blackinton J, Thomas KJ, Ahmad R, Miller DW, Kesavapany S, Singleton A, Lees A, Harvey RJ, Harvey K, Cookson MR (2006) Kinase activity is required for the toxic effects of mutant LRRK2/ dardarin. Neurobiol Dis 23:329-341.

Greggio E, Lewis PA, van der Brug MP, Ahmad R, Kaganovich A, Ding J, Beilina A, Baker AK, Cookson MR (2007) Mutations in LRRK2/dardarin associated with Parkinson disease are more toxic than equivalent mutations in the homologous kinase LRRK1. J Neurochem 102:93-102.

Häbig K, Walter M, Poths S, Riess O, Bonin M (2008) RNA interference of LRRK2-microarray expression analysis of a Parkinson's disease key player. Neurogenetics 9:83-94.

Hamamichi S, Rivas RN, Knight AL, Cao S, Caldwell KA, Caldwell GA (2008) Hypothesis-based RNAi screening identifies neuroprotective genes in a Parkinson's disease model. Proc Natl Acad Sci USA 105:728-733.

Imai Y, Gehrke S, Wang HQ, Takahashi R, Hasegawa K, Oota E, Lu B (2008) Phosphorylation of 4E-BP by LRRK2 affects the maintenance of dopaminergic neurons in Drosophila. EMBO J 27:2432-2443.

Kamath RS, Martinez-Campos M, Zipperlen P, Fraser AG, Ahringer J (2001) Effectiveness of specific RNA-mediated interference through ingested double-stranded RNA in Caenorhabditis elegans. Genome Biol 2:RESEARCH0002.

Kuwahara T, Koyama A, Gengyo-Ando K, Masuda M, Kowa H, Tsunoda M, Mitani S, Iwatsubo T (2006) Familial Parkinson mutant alphasynuclein causes dopamine neuron dysfunction in transgenic Caenorhabditis elegans. J Biol Chem 281:334-340.

Lee SB, Kim W, Lee S, Chung J (2007) Loss of LRRK2/PARK8 induces degeneration of dopaminergic neurons in Drosophila. Biochem Biophys Res Commun 358:534-539.

Lesage S, Dürr A, Tazir M, Lohmann E, Leutenegger AL, Janin S, Pollak P, Brice A (2006) LRRK2 G2019S as a cause of Parkinson's disease in North African Arabs. N Engl J Med 354:422-423.

Li X, Sun AY (1999) Paraquat induced activation of transcription factor AP-1 and apoptosis in PC12 cells. J Neural Transm 106:1-21.

Li Y, Liu W, Oo TF, Wang L, Tang Y, Jackson-Lewis V, Zhou C, Geghman K, Bogdanov M, Przedborski S, Beal MF, Burke RE, Li C (2009) Mutant LRRK2(R1441G) BAC transgenic mice recapitulate cardinal features of Parkinson's disease. Nat Neurosci 12:826-828.

Livak KJ, Schmittgen TD (2001) Analysis of relative gene expression data using real-time quantitative PCR and the 2(-Delta Delta C(T)) Method. Methods 25:402-408.

MacLeod D, Dowman J, Hammond R, Leete T, Inoue K, Abeliovich A (2006) The familial parkinsonism gene LRRK2 regulates neurite process morphology. Neuron 52:587-593.

Moore DJ, Zhang L, Troncoso J, Lee MK, Hattori N, Mizuno Y, Dawson TM, Dawson VL (2005) Association of DJ-1 and parkin mediated by pathogenic DJ-1 mutations and oxidative stress. Hum Mol Genet 14:71-84.

Mosharov EV, Larsen KE, Kanter E, Phillips KA, Wilson K, Schmitz Y, Krantz DE, Kobayashi K, Edwards RH, Sulzer D (2009) Interplay between cytosolic dopamine, calcium, and alpha-synuclein causes selective death of substantia nigra neurons. Neuron 62:218-229.

Nass R, Hall DH, Miller DM 3rd, Blakely RD (2002) Neurotoxin-induced degeneration of dopamine neurons in Caenorhabditis elegans. Proc Natl Acad Sci U S A 99:3264-3269.

Ozelius LJ, Senthil G, Saunders-Pullman R, Ohmann E, Deligtisch A, Tagliati M, Hunt AL, Klein C, Henick B, Hailpern SM, Lipton RB, Soto-Valencia J, Risch N, Bressman SB (2006) LRRK2 G2019S as a cause of Parkinson's disease in Ashkenazi Jews. N Engl J Med 354:424-425.

Paisán-Ruíz C, Jain S, Evans EW, Gilks WP, Simón J, van der Brug M, López de Munain A, Aparicio S, Gil AM, Khan N, Johnson J, Martinez JR,
Nicholl D, Carrera IM, Pena AS, de Silva R, Lees A, Martí-Massó JF, Pérez-Tur J, Wood NW, Singleton AB (2004) Cloning of the gene containing mutations that cause PARK8-linked Parkinson's disease. Neuron 44:595-600.

Plun-Favreau H, Klupsch K, Moisoi N, Gandhi S, Kjaer S, Frith D, Harvey K, Deas E, Harvey RJ, McDonald N, Wood NW, Martins LM, Downward J (2007) The mitochondrial protease HtrA2 is regulated by Parkinson's disease-associated kinase PINK1. Nat Cell Biol 9:1243-1252.

Ren Y, Zhao J, Feng J (2003) Parkin binds to $\alpha / \beta$ tubulin and increases their ubiquitination and degradation. J Neurosci 23:3316-3324.

Ren Y, Jiang H, Yang F, Nakaso K, Feng J (2009) Parkin protects dopaminergic neurons against microtubule-depolymerizing toxins by attenuating microtubule-associated protein kinase activation. J Biol Chem 284:4009-4017.

Sakaguchi-Nakashima A, Meir JY, Jin Y, Matsumoto K, Hisamoto N (2007) LRK-1, a C. elegans PARK8-related kinase, regulates axonal-dendritic polarity of SV proteins. Curr Biol 17:592-598.

Schapira AH (2008) Mitochondria in the aetiology and pathogenesis of Parkinson's disease. Lancet Neurol 7:97-109.

Shioi G, Shoji M, Nakamura M, Ishihara T, Katsura I, Fujisawa H, Takagi S (2001) Mutations affecting nerve attachment of Caenorhabditis elegans. Genetics 157:1611-1622.

Smith WW, Pei Z, Jiang H, Dawson VL, Dawson TM, Ross CA (2006) Kinase activity of mutant LRRK2 mediates neuronal toxicity. Nat Neurosci 9:1231-1233.

Suzuki H, Kerr R, Bianchi L, Frøkjaer-Jensen C, Slone D, Xue J, Gerstbrein B, Driscoll M, Schafer WR (2003) In vivo imaging of C. elegans mechanosensory neurons demonstrates a specific role for the MEC-4 channel in the process of gentle touch sensation. Neuron 39:1005-1017.

Valente EM, Abou-Sleiman PM, Caputo V, Muqit MM, Harvey K, Gispert S, Ali Z, Del Turco D, Bentivoglio AR, Healy DG, Albanese A, Nussbaum R, González-Maldonado R, Deller T, Salvi S, Cortelli P, Gilks WP, Latchman DS, Harvey RJ, Dallapiccola B, Auburger G, Wood NW (2004) Hereditary early-onset Parkinson's disease caused by mutations in PINK1. Science 304:1158-1160.

Ved R, Saha S, Westlund B, Perier C, Burnam L, Sluder A, Hoener M, Rodrigues CM, Alfonso A, Steer C, Liu L, Przedborski S, Wolozin B (2005) Similar patterns of mitochondrial vulnerability and rescue induced by genetic modification of $\alpha$-synuclein, parkin and DJ-1 in Caenorhabditis elegans. J Biol Chem 280:42655-42668.

West AB, Moore DJ, Biskup S, Bugayenko A, Smith WW, Ross CA, Dawson VL, Dawson TM (2005) Parkinson's disease-associated mutations in leucine-rich repeat kinase 2 augment kinase activity. Proc Natl Acad Sci U S A 102:16842-16847.

West AB, Moore DJ, Choi C, Andrabi SA, Li X, Dikeman D, Biskup S, Zhang Z, Lim KL, Dawson VL, Dawson TM (2007) Parkinson's diseaseassociated mutations in LRRK2 link enhanced GTP-binding and kinase activities to neuronal toxicity. Hum Mol Genet 16:223-232.

Wood WB (1988) The nematode Caenorhabditis elegans. Cold Spring Harbor, NY: Cold Spring Harbor Laboratory.

$\mathrm{Xu} \mathrm{K}$, Tavernarakis N, Driscoll M (2001) Necrotic cell death in C. elegans requires the function of calreticulin and regulators of $\mathrm{Ca}^{2+}$ release from the endoplasmic reticulum. Neuron 31:957-971.

Yamada K, Fukushima T (1993) Mechanism of cytotoxicity of paraquat. II. Organ specificity of paraquat-stimulated lipid peroxidation in the inner membrane of mitochondria. Exp Toxicol Pathol 45:375-380.

Yang Y, Gehrke S, Imai Y, Huang Z, Ouyang Y, Wang JW, Yang L, Beal MF, Vogel H, Lu B (2006) Mitochondrial pathology and muscle and dopaminergic neuron degeneration caused by inactivation of Drosophila Pink1 is rescued by Parkin. Proc Natl Acad Sci U S A 103:10793-10798.

Zimprich A, Biskup S, Leitner P, Lichtner P, Farrer M, Lincoln S, Kachergus J, Hulihan M, Uitti RJ, Calne DB, Stoessl AJ, Pfeiffer RF, Patenge N, Carbajal IC, Vieregge P, Asmus F, Müller-Myhsok B, Dickson DW, Meitinger T, Strom TM, Wszolek ZK, Gasser T (2004) Mutations in LRRK2 cause autosomal-dominant parkinsonism with pleomorphic pathology. Neuron 44:601-607. 